Article

\title{
Temperature Dependence of Oxidation Kinetics of Extra Virgin Olive Oil (EVOO) and Shelf-Life Prediction
}

\author{
Lanfranco Conte $^{1}$, Andrea Milani ${ }^{1}{ }^{\circledR}$, Sonia Calligaris ${ }^{1}$, Pierangela Rovellini ${ }^{2}{ }^{\circledR}$, Paolo Lucci ${ }^{1, *}$ \\ and Maria Cristina Nicoli ${ }^{1}$ \\ 1 Department of Agri-Food, Animal and Environmental Sciences, University of Udine, via Sondrio 2/a, \\ 33100 Udine, Italy; lanfranco.conte@uniud.it (L.C.); milani.andrea.1@spes.uniud.it (A.M.); \\ sonia.calligaris@uniud.it (S.C.); mariacristina.nicoli@uniud.it (M.C.N.) \\ 2 Innovhub Stazioni Sperimentali per l'Industria s.r.l., Via Giuseppe Colombo 79, 20133 Milan, Italy; \\ pierangela.rovellini@mi.camcom.it \\ * Correspondence: paolo.lucci@uniud.it; Tel.: +39-0432-55817
}

Received: 10 February 2020; Accepted: 4 March 2020; Published: 5 March 2020

\begin{abstract}
Producers have to guarantee the extra virgin olive oil (EVOO) quality characteristics reported in the Regulation (CEE) 2568/91 throughout the product shelf-life (SL). Unfortunately, due to the development of oxidative reactions, some quality indices change during storage leading to a progressive deterioration of EVOO quality. To avoid the risk of product downgrading in the virgin oil category, the development of effective shelf-life prediction models is extremely important for the olive oil industry. In this research, the accelerated shelf-life testing (ASLT) protocol was applied to evaluate the temperature dependence of selected oxidation indexes as well as to develop a shelf-life predictive model. The evolution of conventional (peroxide value, K232, K270, polyphenols, tocopherols and hexanal) and unconventional parameters (conjugated trienes and pyropheophytin $a$ ) was monitored in bottled EVOO stored in the dark at increasing temperature $\left(25,40,50\right.$ and $\left.60^{\circ} \mathrm{C}\right)$. Accordingly, for well-packed products with reduced oxygen in headspace, the best shelf-life index allowing the ability to predict EVOO SL turned out to be K270. In addition, pyropheophytin $a(\%)$ has been shown to be more sensitive to temperature changes than the secondary oxidation indices, thus suggesting its use as a freshness indicator for storage temperatures higher than $25^{\circ} \mathrm{C}$.
\end{abstract}

Keywords: extra virgin olive oil; shelf-life; consumers; oil producers; accelerated shelf-life test; olive oil stability; prediction model

\section{Introduction}

Extra virgin olive oil (EVOO) traditionally represents the major edible vegetable oil consumed in Mediterranean countries. However, today it is globally recognized and appreciated by consumers in non-producing countries. This is due to its unique sensory characteristics combined with the well-demonstrated health-promoting capacity. The latter is mainly associated with a high level of monounsaturated fatty acids (mostly oleic acid) and minor components, such as phenolic compounds and tocopherols [1]. Based on EU Regulation (CEE) 2568/91 as well as International Olive Oil Council (IOC) trade standard [2], the oil extracted from olives by mechanical methods must comply with a number of quality indices to be classified in the extra virgin category [3]. It is a matter of fact that the compliance with these parameters must be guaranteed throughout the product shelf-life to avoid the risk of the product downgrading in the virgin oil category. This situation could be commercially dangerous for producers with possible negative impact on brand reputation. As is well known, many EVOO quality indicators could sharply change during product storage due to the development of 
oxidative reactions. Typical examples are peroxide number, absorbance values in UV region at 232 and $270 \mathrm{~nm}$ and the sensory profile [4]. Beside these compulsory indexes, other indicators, mainly related to oil freshness profile, such as phenol, tocopherol and pigments content, could markedly change during product storage $[5,6]$. Therefore, the possibility to predict the time needed to exceed the regulatory limits for the EVOO category could be particularly helpful to define the EVOO shelf-life (SL) and thus the "best before" date to be reported on the label based on Regulation EU 1169/2015 [7].

As summarized by Nicoli [8], the SL of a packed food product is the length of time after production during which the product retains a specified quality level under well-defined conditions.

A shelf-life study can be divided into three fundamental steps. The first step implies the identification of the most critical chemical, physical or biological event leading to product quality depletion, followed by the definition of the relevant acceptability limit. Considering EVOO, as previously reported, oxidation is expected to be the main quality deterioration mechanism during storage. Oxidation is usually monitored by a number of indicators, moving from very simple and cheap methodologies to more complex and costly analytical procedures. At present in EU the only available acceptability limits for EVOO are those provided by law (i.e., peroxide value, K232 and K270). Table 1 shows the maximum level of these index allowed by Regulation EC 2568/91. Other early indicators able to monitor product susceptibility to oxidation may also be considered as alternatives or in place of the compulsory ones. However, in this case, the availability of acceptability limits is essential to go forward in a shelf-life study.

Table 1. Initial composition and characteristics of the studied extra virgin olive oil (EVOO).

\begin{tabular}{ccc}
\hline Qualitative Characteristics & Values & IOC Reference Values $[2,3]$ \\
\hline $\mathrm{PV}\left(\mathrm{meqO}_{2} / \mathrm{kg}\right)$ & 5.7 & 20.0 \\
$\mathrm{~K} 232(\mathrm{ex}, 1 \%, 1 \mathrm{~cm})$ & 1.81 & 2.50 \\
$\mathrm{~K} 270(\mathrm{ex}, 1 \%, 1 \mathrm{~cm})$ & 0.15 & 0.22 \\
total tyrosol and hydroxytyrosol $(\mathrm{mg} / \mathrm{kg})$ & 332.9 & n.c. \\
$\alpha$-tocopherol $(\mathrm{mg} / \mathrm{kg})$ & 205.6 & n.c. \\
$\beta+\delta$-tocopherol $(\mathrm{mg} / \mathrm{kg})$ & 15.6 & n.c. \\
Y-tocopherol $(\mathrm{mg} / \mathrm{kg})$ & 4.3 & n.c. \\
total tocopherols $(\mathrm{mg} / \mathrm{kg})$ & 225.6 & n.c. \\
chlorophyllics pigments $(\mathrm{mg} / \mathrm{kg})$ & 23.5 & n.c. \\
\hline Main Fatty Acids $(\%)$ & & $7.50-20.00$ \\
$\mathrm{C} 16: 0$ & 10.7 & $0.50-5.00$ \\
$\mathrm{C} 18: 0$ & 1.9 & \\
$\mathrm{C} 18: 1 \Delta 9 \mathrm{c}$ & 76.5 & $55.00-83.00 *$ \\
$\mathrm{C} 18: 1 \Delta 11 \mathrm{c}$ & 1.2 & $2.50-21.00$ \\
$\mathrm{C} 18: 2^{\Delta 9 \mathrm{c}, 12 \mathrm{c}}$ & 7.8 & $\leq 1.00$ \\
$\mathrm{C} 18: 3^{\Delta 9 \mathrm{c}, 12 \mathrm{c}, 15 \mathrm{c}}$ & 0.6 & -
\end{tabular}

Legend for fatty acids- $m: n \Delta \mathrm{x}, m=$ number of carbon atoms, $n=$ number of double bonds, $\mathrm{x}=$ position of double bonds; n.c.: not considered; ${ }^{*} \sum \mathrm{C} 18: 1^{\Delta 9}$ cis $+C 18: 1^{\Delta 11}$ cis; IOC: International Olive Oil Council. PV: peroxide value.

In the next step of the SL assessment process, the changes of the selected quality indicators are monitored as a function of time under storage conditions mimicking the foreseeable ones (real-time shelf-life testing) or under environmental conditions able to speed up the deteriorative events (accelerated shelf-life testing-ASLT). Finally, data should be modeled to obtain a shelf-life estimation or prediction, respectively $[9,10]$. Among all environmental factors potentially applicable to accelerate oxidative reactions, temperature is certainly the most widely used. This is not only due to the fact that temperature is one of the most critical factors affecting food reaction kinetics, but also thanks to the 
availability of a mathematical description of the temperature sensitivity of quality loss rates, that is the well-known Arrhenius equation, Equation (1) [11]:

$$
k=k_{0} \cdot e^{-\frac{E_{a}}{R T}}
$$

where $k$ is the reaction rate constant; $R$ is the molar gas constant $\left(8.31 \mathrm{~J} \mathrm{~K}^{-1} \mathrm{~mol}^{-1}\right), T$ is the absolute temperature $(\mathrm{K}) ; E_{a}$ is the apparent activation energy $\left(\mathrm{J} \mathrm{mol}^{-1}\right)$ and $k_{0}$ is the so-called pre-exponential factor. Even if this model has been developed theoretically on the molecular basis for reversible chemical reactions, it has been shown to hold empirically for estimating the rate of a wide range of complex chemical, physical and sensory changes occurring in foods $[12,13]$. Based on this equation, the temperature sensitivity of deteriorative reactions can be described by the activation energy value $\left(E_{a}\right)$. In the case of lipid oxidation, the range of $E_{a}$ values moves from about 20 to $150 \mathrm{~kJ} / \mathrm{mol}$ [10]. This wide range of $E_{a}$ magnitudes obviously depends on the different food characteristics, processing and storage conditions. For EVOO, Mancebo-Campos et al. [14] reported the temperature dependence from 25 to $60^{\circ} \mathrm{C}$ of oxidative reactions by means of the Arrhenius equation, highlighting $E_{a}$ values around $65 \mathrm{~kJ} / \mathrm{mol}$ for primary oxidation products and of about $77 \mathrm{~kJ} / \mathrm{mol}$ for secondary oxidation products. Besides, Calligaris et al. [15] reported, in the same temperature range, $E_{a}$ values of 42 and $33 \mathrm{~kJ} / \mathrm{mol}$ for peroxide value and hexanal, respectively. It should be highlighted that in both cases the samples were stored under a high oxygen environment, which is completely different from the actual exposure conditions of EVOO during storage on the market.

Finally, it should be also stressed that other studies reported the temperature sensitivity of oxidation obtained at elevated temperatures. As stated by Frankel [16] for food lipids, the use of temperatures higher than $60^{\circ} \mathrm{C}$ for vegetable oils is questionable, because samples develop excessive rancidity levels, which are not relevant to what happens under normal storage conditions. In agreement with this observation, some accelerated tests developed to evaluate the oxidative stability of oils in a short time (e.g., Rancimat and OSI) showed a low correlation compared with tests conducted under actual storage conditions [10,17].

Based on these considerations, the olive oil industry is still in great need of effective shelf-life prediction models based on simple analytical descriptors to be employed for ensuring the product quality during storage on the market. The aim of this paper is to apply the ASLT protocol to evaluate the temperature dependence of different oxidation indexes as well as to develop a shelf-life predictive model. To this purpose, a selected EVOO oil was bottled mimicking commercial conditions and stored at increasing temperature in the dark $\left(25,40,50\right.$ and $\left.60^{\circ} \mathrm{C}\right)$. During storage conventional (peroxide value, K232, K270, polyphenols, tocopherols and hexanal) and unconventional parameters (conjugated trienes and pyropheophytin $a$ ) were monitored and relevant oxidation rates were calculated. Rate constants were then modeled to define the temperature dependence, and finally to develop a shelf-life prediction model.

\section{Materials and Methods}

\subsection{Chemicals}

Acetone, acetonitrile, isopropanol, ethanol, methanol and $n$-hexane (all HPLC grade) were purchased from Sigma-Aldrich (Milano, Italia). Water was purified with a Milli-Q system (Millipore, Bedford, MA, USA). All other reagents were of analytical grade. Tocopherol $(\alpha, \gamma$ and $\delta$-tocopherols) and phenolic acids (tyrosol and hydroxytyrosol) standards were purchased from Sigma-Aldrich (Milano, Italia). Chlorophyll A was purchased from Sigma-Aldrich (Milano, Italia). 


\subsection{Olive Oil Samples}

Extra virgin olive oil (EVOO) (Olea europaea L., cv Coratina) samples were taken from a homogeneous batch produced in 2018. Samples were kindly provided by Castel del Chianti (Tavarnelle Val di Pesa, Firenze, Italy).

\subsection{Storage Conditions}

Aliquots of $250 \mathrm{~mL}$ of EVOO were commercially packed in clear glass bottles with a metal cap and PTFE internal septum. A total of 60 bottles (15 for each selected temperature) were stored at $25,40,50$ and $60^{\circ} \mathrm{C}$ in the dark in incubators (FTC 90I Refrigerated Incubator, Monza, Italy) for up to 300 days. At different storage times, one bottle was taken from the incubators and subjected to analytical determinations.

\subsection{Reference Chemical Analysis}

Determination of acidity as oleic acid, peroxide value (PV), specific UV absorption at $232 \mathrm{~nm}$ (K232) and $270 \mathrm{~nm}(\mathrm{~K} 270)$ and fatty acids methyl esters were conducted according to the procedure reported in Regulation EC 2568/91 and its amendments [2]. Table 1 gives the initial fatty acid composition of EVOO.

\subsection{Phenolic Compounds}

Phenolic compounds were extracted following the International Oleic Council method [18] and hydrolyzed according to the method proposed by Rovellini et al. [19]. The hydrolyzed sample was then analyzed by UHPLC using an Agilent Poroshell 120 EC-C18 reversed-phase column $(2.7 \mu \mathrm{m}$ particle size, $4.6 \times 150 \mathrm{~mm}$ ) on a Shimadzu Nexera UHPLC System (Shimadzu Nexera, Kyoto, Japan) equipped with dual pump LC-30AD, on-line degasser DGU-20AS, column oven CTO-30A, autosampler SIL-30AC and diode array detector (SPD-M20A). Gradient separation was created from solvent A (water with $2 \%$ of acetic acid) and solvent B (acetonitrile) as follows: starting from 95\% A; 0.01-12 min linear gradient from $5 \%$ to $70 \% \mathrm{~B} ; 12-13$ min linear gradient from $70 \%$ to $90 \% \mathrm{~B}$; isocratic condition kept up to $17 \mathrm{~min} ; 17 \mathrm{~min}$ back to initial condition at $5 \% \mathrm{~B}$; isocratic step kept up to $22 \mathrm{~min}$ for column re-conditioning. The mobile phase flow rate was $450 \mu \mathrm{L} \mathrm{min}^{-1}$. The column temperature was $30{ }^{\circ} \mathrm{C}$. Injected volumes for each sample was $5 \mu \mathrm{L}$. The detector was set at $280 \mathrm{~nm}$. Polyphenols quantification was obtained using calibration curves obtained by injection on the column of different amounts of both tyrosol and hydroxytyrosol (10-600 ng) with $R^{2}$ values higher than 0.999 , in all cases.

\subsection{Tocopherols}

UHPLC analysis was realized using a Shimadzu Nexera (Shimadzu, Kyoto, Japan) coupled with the same components used for polyphenols analysis and a fluorescence detector RF-20Axs with double acquisition channels and a $12 \mu \mathrm{L}$ cell. The detector was set at $296 \mathrm{~nm}$ and $325 \mathrm{~nm}$ for exciting and emission wavelengths, respectively. Oil samples were diluted in 2-propanol for reaching a $100 \mathrm{mg} / \mathrm{mL}$ concentration and $1 \mu \mathrm{L}$ injected on the column as a compromise between sensibility and column capacity.

The chromatographic separation was performed using an Agilent Eclipse PAH column $(1.8 \mu \mathrm{m}$ particle size, $4.6 \times 50 \mathrm{~mm}$ ) under isocratic conditions with solvent A (methanol) and B (acetonitrile) in the ratio $60 / 40(v / v)$ and a total flow of $600 \mu \mathrm{L} \mathrm{min}{ }^{-1}$. The oven temperature was set to $30{ }^{\circ} \mathrm{C}$. The injected volume for each sample was $1 \mu \mathrm{L}$.

Tocopherols were quantified using a calibration curve for $\alpha, \beta+\gamma$ and $\delta$ respectively in the range $0.05-100 \mathrm{ng}$ on the column with $R^{2}$ values higher than 0.999 . 


\subsection{Pyropheophytin a (ISO 29841:2009 (E))}

Pyropheophytin $a$ was measured using method ISO 29841:2009 [20]. To isolate pigments was used an SPE SiOH column 6 mL/1 g (Chromabond Macherey-Nagel GmbH \& Co, Düren, Germany) using the first $10 \mathrm{~mL}$ of a petroleum ether/ethyl ether solution in the ratio 90:10 for the elution of non-polar compounds than $10 \mathrm{~mL}$ of acetone as elution solvent for chlorophylls fraction. The eluate was then analyzed by reverse-phase Spherisorb ODS2 C18-HPLC and the separated components were monitored at $410 \mathrm{~nm}$ using a photometric detector. The results were expressed as relative proportions (pyropheophytin $a$, \%PP) of the analyses (pyropheophytin $a$ and pheophytin $a$ and $a^{\prime}$ ), in relation to the sum of pyropheophytin $a$ and pheophytin $a+a^{\prime}$.

\subsection{Conjugated Trienes}

The analysis of conjugated trienes (CT) was performed following the method proposed by Rovellini and Cortesi [21]. Briefly, the sample was diluted in $1 \mathrm{~mL}$ of isopropanol and then analyzed in HPLC/DAD instrument (Finnigan P4000 HPLC, Saint Hose, CA USA), injecting $20 \mu \mathrm{L}$. Conjugated benzylester fatty acids derivatives were separated using a RP18 Spherisorb ODS2 column (5 $\mu \mathrm{m}$ particle size, $4 \times 250 \mathrm{~mm}$ ) using a binary solvent system of water and acetonitrile, starting with a $50 \%$ of solvent B increased to $100 \%$ in the first $50 \mathrm{~min}$, maintained for $15 \mathrm{~min}$ and then reverted to $50 \%$ at a flow rate of $1000 \mu \mathrm{L} \mathrm{min}{ }^{-1}$. Chromatograms were recorded at $255 \mathrm{~nm}$ and a scan spectra acquisition from 200 to $400 \mathrm{~nm}$ is necessary to identify al peaks.

\subsection{Volatile Compound Analysis}

Volatile compounds considered in this work are hexanal, octane, nonanal, heptadienal and decadienal that were quantified following the method proposed by Vichi [22].

As an internal standard, a solution of 4-methyl-2-pentanol dissolved in refined oil at the concentration of $45 \mu \mathrm{g} / \mathrm{g}$ was used (Sigma Aldrich, St. Louis, MO, USA). Extraction was performed through an SPME fiber with DVB-Carboxen_PDMS 50/30 $\mu \mathrm{m}$ phase $2 \mathrm{~cm}$ long (Agilent Technologies, Santa Clara, CA, USA). The samples were prepared by weighing $2.0 \mathrm{~g}$ of sample and adding $50 \mu \mathrm{L}$ of an internal standard solution in a $10 \mathrm{~mL}$ vial closed with a metallic screw cap and PTFE/silicon septum (Agilent Technologies). The sample was equilibrated for $20 \mathrm{~min}$ at $40^{\circ} \mathrm{C}$. After the equilibration, a DVB-Carboxen_PDMS 50/30 $\mu \mathrm{m}$ phase $2 \mathrm{~cm}$ long SPME fiber (Agilent Technologies, Santa Clara, CA, USA) was exposed to the head-space (HS) for $30 \mathrm{~min}$ at $40^{\circ} \mathrm{C}$. Chromatographic analysis was then performed using a GCMS 5977A Extractor Source (Agilent Technologies, Santa Clara, CA), with autosampler CTC for SPME injection with a VF-WAX column $(30 \mathrm{~m} \times 0.25 \mathrm{~mm}$ I.D. $\times 0.25 \mu \mathrm{m})$ (Agilent Technologies). The analytes were desorbed to the hot injection port of GC for $2 \mathrm{~min}$ at $250{ }^{\circ} \mathrm{C}$ in a splitless mode. Oven temperature is kept at $40^{\circ} \mathrm{C}$ for $10 \mathrm{~min}$, then increased $3{ }^{\circ} \mathrm{C} / \mathrm{min}$ up to $200{ }^{\circ} \mathrm{C}$ and kept for $2 \mathrm{~min}$. Helium was used as a carrier gas at a constant flow of $1 \mathrm{~mL} / \mathrm{min}$. Temperatures of transfer line, ionic source and quadrupole were 280,175 and $150{ }^{\circ} \mathrm{C}$, respectively. Ionization energy was fixed at $70 \mathrm{eV}$ with an acquisition range of $40-350 \mathrm{~m} / \mathrm{z}$. Integration and identification were carried out using Agilent Mass Hunter Qualitative Analysis B.06.00 software (Agilent Technologies) with a deconvolution algorithm and NIST 14 library and linear retention indexes.

\subsection{Kinetics Data Analysis}

Apparent zero-order rate constants $(k)$ of oxidation indexes as a function of storage time were calculated by linear regression. No lag phase was detected. Only the increasing part of the curves was considered.

The effect of temperature on the rate of lipid oxidation was evaluated by means of the Arrhenius equation. To make a better estimation of the apparent activation energy a one-step non-linear regression was applied to all data by using the reparametrized Arrhenius equation, in which it was inserted a 
reference temperature chosen in the middle of the temperature range considered in the experimental plan, Equation (2)

$$
\ln k=\ln k_{r e f}-\frac{E_{a}}{R}\left(\frac{1}{T}-\frac{1}{T_{r e f}}\right)
$$

where $k$ is the apparent reaction rate, $R$ is the molar gas constant $(8.31 \mathrm{~J} / \mathrm{K} / \mathrm{mol}), T$ is the absolute temperature $(\mathrm{K})$, and $k_{\text {ref }}$ is the apparent reaction rate at $T_{\text {ref }}\left(45^{\circ} \mathrm{C}\right) . \mathrm{T}_{\text {ref }}$ was chosen as $318 \mathrm{~K}$, which is the central value of the temperature interval considered in the study. $E_{a}$ and $k_{r e f}$ were determined by linear regression analysis and used to calculate $k_{0}$, Equation (3):

$$
k_{0}=e^{\left(\ln k_{r e f}+\frac{E_{a}}{R T_{r e f}}\right)}
$$

\subsection{Statistical Analysis}

Data were expressed as the mean and standard deviation of at least two analytical determinations on two replicated samples. Statistical elaboration was performed with R Software (3.2.2 version, R Project for Statistical Computing; The R Foundation for Statistical Computing, Wien, Austria). Bartlett's test was used to check the homogeneity of variance, one-way ANOVA was carried out and Tukey's HSD test was used as a post-hoc test to determine statistically significant differences among means $(p<0.05)$. Linear regression analysis was performed using Microsoft Excel 2016 (Microsoft Corp., Redmond, WA, USA). The goodness of fitting was evaluated by using the coefficient of determination $\left(R^{2}\right)$, the standard error (SE) and the $p$-value $(p)$.

\section{Results}

\subsection{EVOO Initial Chemical Composition}

The chemical characteristics of olive oil studied are presented in Table 1. According to EVOO limits established by IOC for quality indexes and EU regulation [2], our matrix was classified as extra virgin olive oil. EVOO sample was also characterized by a high level of total tocopherols $(225.6 \mathrm{mg} / \mathrm{kg})$ and hydroxytyrosol and its derivatives content after acidic hydrolytic procedure $(332.9 \mathrm{mg} / \mathrm{kg}$ ), thus over the minimum content fixed by the European Food Safety Authority for the health claim on "olive oil polyphenols" (Commission Regulation (EU) 432/2012) (250 mg/kg) [23].

\subsection{Kinetics of Quality Indicators during Storage at Increasing Temperatures}

The development of oxidative reactions in EVOO during storage was monitored at 25, 40, 50 and $60{ }^{\circ} \mathrm{C}$ by following the changes of the following parameters: peroxide value, K232, K270, polyphenols, tocopherols, conjugated trienes, hexanal and pyropheophytins.

Some of these selected indices did not show any relevant changes during storage even at the highest temperatures. In particular, neither PV nor ultraviolet coefficient K232 demonstrated a significant increase over time (Supplementary Tables S1-S4). This result highlights that, in the experimental condition tested, primary oxidation products did not further develop during storage without reaching the compulsory limits of $20 \mathrm{meqO}_{2} / \mathrm{Kg}$ and 2.50 absorbency for PV and $\mathrm{K} 232$, respectively. As well known, during oxidation hydroperoxides, as intermediate reaction products, could at the same time be formed and decomposed and the behavior of the relevant analytical indexes is due to the concomitant development of both events. When the rate of formation is higher than that of decomposition, an increase in both PV and K232 is expected. On the other hand, when the decomposition rate prevails, the value of both indices decreases. Thus, when no changes of the primary oxidation indicators are observed, two different situations could be hypothesized: the reaction in the observed time did not proceed or the formation and decomposition rate of hydroperoxides are similar. Our results are also consistent with those found by Brenes et al. [24], who observed negligible changes of the PV and K232 throughout the storage period ( 1 year at $30^{\circ} \mathrm{C}$ ) of oil bottled in closed amber glass jars with reduced 
headspace. On the contrary, Mancebo-Campos et al. [14] revealed a progressive increase of PV and K232 during storage at different temperatures, similarly to data shown by Gómez-Alonso et al. [25] and Calligaris et al. [15]. It should be noted, however, that in these studies samples were stored in the dark in open containers or in the ordinary atmosphere and, thus, under a completely different condition compared to that considered in our study, in which the oxygen content in the container can be assumed as the limiting reactant.

To better understand the evolution of oxidation, the parameter $\mathrm{K} 270$ was monitored as an indicator of the secondary oxidation (Figure 1a). A significant increase during the storage of this index was denoted. This result was further confirmed by the increase of conjugated trienes content and hexanal (Figure $1 \mathrm{~b}, \mathrm{c}$ ). In fact, $\mathrm{k} 270$ which is considered an index of conjugated trienes, could also be influenced by other compounds present in olive oil that could interfere with this measure, such as phenols and other derivatives. On the other hand, the measurement of conjugated trienes by HPLC is specific for the quantification of this class of compounds that derives from the reduction of hydroperoxides of linoleic acid and that can be considered secondary oxidized products. As expected, the rise of the storage temperatures accelerated the changes in these indexes. These findings are in agreement with previous studies $[24,26]$.

(a)

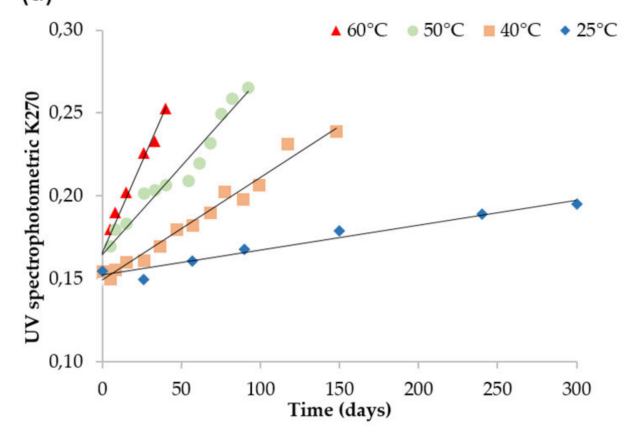

(b)

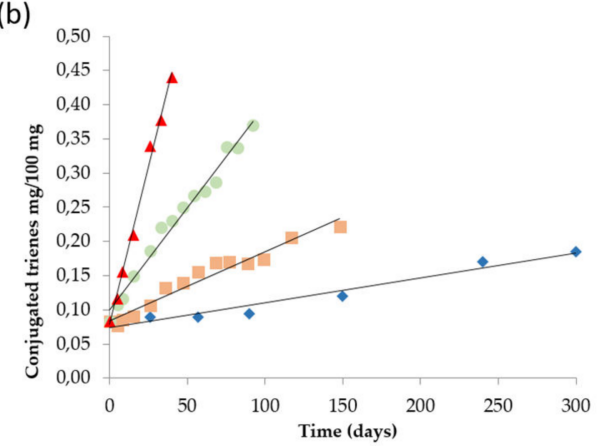

(c)

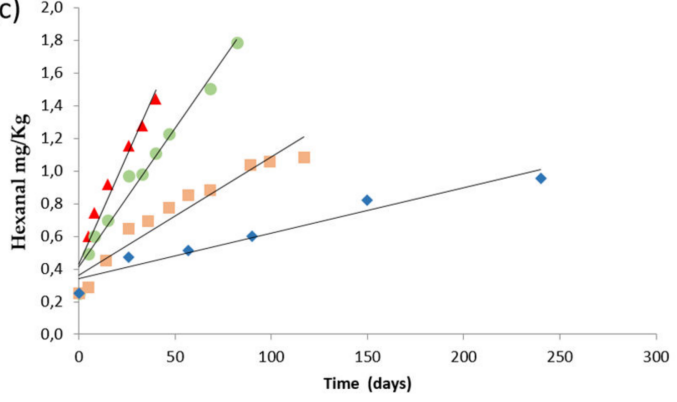

Figure 1. Changes of K270 (a), conjugate trienes (b) and hexanal (c) of extra virgin olive oil stored at 25, 40,50 and $60{ }^{\circ} \mathrm{C}$ (symbols: experimental data, solid line: regression results).

Besides these quality indices, the changes of pyropheophytin $a$ during storage was also monitored. Pyropheophytins in olive oil are formed due to degradations of chlorophyll pigments and this reaction begins soon after the oil is extracted. The pigments break down due to a process that involves the decarbomethoxylation of chlorophyll and pheophytins to form pyropheophytins [6]. Figure 2 shows the kinetics of pyropheophytin $a$ formation as a function of storage time at $25,40,50$, and $60^{\circ} \mathrm{C}$. After a first sharp linear increase of this index over time, \%PPP reached a plateau at the highest temperatures.

Interestingly, the main antioxidants present in EVOO did not participate in these events as demonstrated by their evolution over time (Supplementary Tables S1-S4). In fact, both total polyphenols and total tocopherols did not show any significant changes upon storage independently on storage temperature. 


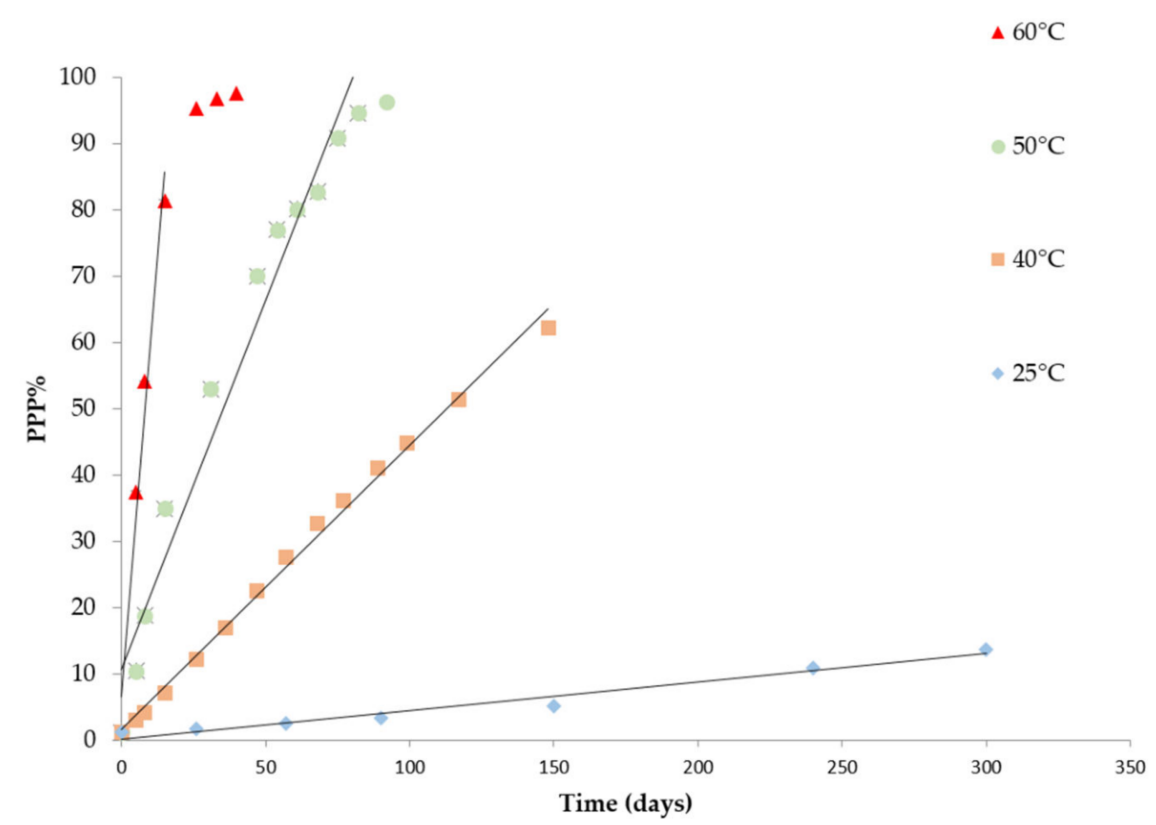

Figure 2. Changes of \%PPP of extra virgin olive oil stored at 25, 4050 and $60{ }^{\circ} \mathrm{C}$ (symbols: experimental data, solid line: regression results).

\subsection{Modeling the Temperature Dependence of the Oxidation Rate}

Based on the results above described, K270, conjugated trienes, hexanal and \%PPP resulted in good indicators of product quality depletion during storage at different temperatures. In the attempt to develop a predictive shelf-life model it is fundamental to define the temperature dependence of the rates of these indexes. Considering the data above reported, all the selected indicators followed a pseudo zero reaction order. Thus, the apparent zero-order rate constants were computed by linear regression analysis of the increasing part of the curves reported in Figures 1 and 2. Table 2 shows the values of the rate constants $(k)$, the relevant standard error and the coefficient of determination. In all cases, this reaction order well described the evolution of the selected indexes $\left(R^{2}>0.95 ; p<0.05\right)$.

Table 2. Apparent zero-order reaction rate of K270, \%PPP and CT of EVOO stored at 25, 40, 50 and $60^{\circ} \mathrm{C}$.

\begin{tabular}{|c|c|c|c|c|c|c|c|c|}
\hline \multirow[b]{2}{*}{$\mathrm{T}\left({ }^{\circ} \mathrm{C}\right)$} & \multicolumn{2}{|l|}{ K270 } & \multicolumn{2}{|l|}{$\% P P P$} & \multicolumn{2}{|l|}{$\mathrm{CT}$} & \multicolumn{2}{|l|}{ Hexanal } \\
\hline & $\begin{array}{c}k_{270} \\
\text { (D.O.day }^{-1.10^{-3}} \text { ) }\end{array}$ & $R^{2}$ & $\begin{array}{c}k_{P P P}(\% \mathrm{PPP} \\
\left.\text { day }^{-1}\right)\end{array}$ & $R^{2}$ & $\begin{array}{l}k_{C T}(\mathrm{mg} / \mathrm{kg} \\
\left.\text { day }^{-1.10^{-3}}\right)\end{array}$ & $R^{2}$ & $\begin{array}{c}k_{\text {hexanal }}(\mathrm{mg} / \mathrm{kg} \\
\left.\text { day }^{-1} \cdot 10^{-3}\right)\end{array}$ & $R^{2}$ \\
\hline 25 & $0.18 \pm 0.01$ & 0.96 & $0.043 \pm 0.03$ & 0.99 & $0.36 \pm 0.01$ & 0.99 & $2.77 \pm 0.34$ & 0.95 \\
\hline 40 & $0.62 \pm 0.02$ & 0.98 & $0.428 \pm 0.01$ & 0.99 & $1.05 \pm 0.06$ & 0.98 & $7.24 \pm 0.73$ & 0.92 \\
\hline 50 & $1.07 \pm 0.07$ & 0.95 & $1.112 \pm 0.07$ & 0.96 & $3.01 \pm 0.11$ & 0.95 & $16.91 \pm 0.94$ & 0.94 \\
\hline 60 & $2.20 \pm 0.02$ & 0.97 & $5.270 \pm 0.49$ & 0.97 & $9.17 \pm 0.32$ & 0.96 & $26.62 \pm 2.88$ & 0.97 \\
\hline
\end{tabular}

Furthermore, to highlight the temperature dependence of K270, hexanal, conjugated trienes and \%PPP, the values of $k$ reported in Table 2 were plotted according to the Arrhenius model (Figure 3 ). In all cases, the Arrhenius behavior was fulfilled in the entire range of temperatures considered $\left(R^{2}>0.97, p<0.05\right)$ and $E_{a}$ and $k_{0}$ were calculated by using Equations (2) and (3), respectively (Table 3$)$. It should be remembered in this context, oxidation being a complex reaction, that these values cannot provide a mechanistic interpretation of the reaction, but rather can be used as descriptive tools of the temperature dependence of the reaction [27].

Examining the data in Table 3, it can be noted that the highest values of $E_{a}$ and $k_{0}$ were relevant to the changes of \%PPP, highlighting the highest temperature sensitivity of the evolution of this index in comparison to that of the considered secondary oxidation products. 
The experimental $E_{a}$ values acquired were consistent with literature data on lipid oxidation in different matrices and by considering different indices, ranging from 20 to $200 \mathrm{~kJ} / \mathrm{mol}$ [28]. Considering only references on EVOO, very few information can be found on the application of the ASLT approach. In this regard, an accelerated storage test was applied by Mancebo-Campos et al. [14] to evaluate the temperature dependence of oxidation of EVOO. The authors reported $E_{a}$ values of about 65 and $76 \mathrm{~kJ} / \mathrm{mol}$, respectively for primary and secondary oxidation products. However, it should be noted that these authors stored the oil at increasing temperatures from 25 to $60^{\circ} \mathrm{C}$ at a high oxygen concentration. Regarding \%PPP temperature dependence, Aparicio-Ruiz et al. [29] studied the thermal degradation of chlorophyll pigments and the consequent formation kinetics of pyropheophytin $a$ in virgin olive oil (VOO) in a temperature range from 60 to $120^{\circ} \mathrm{C}$ in absence of air. The acquired $E_{a}$ value was consistent with our research data and around $80-100 \mathrm{~kJ} / \mathrm{mol}$. It is well evident the strong impact of the temperature on this index, thus suggesting the use of pyropheophytins as indicator of olive oil quality and freshness, especially if considering that PPP evolution has been proven to be not influenced by the initial quality of the oil, cultivar or growing environments but mainly by storage time, temperature and light exposure [24].

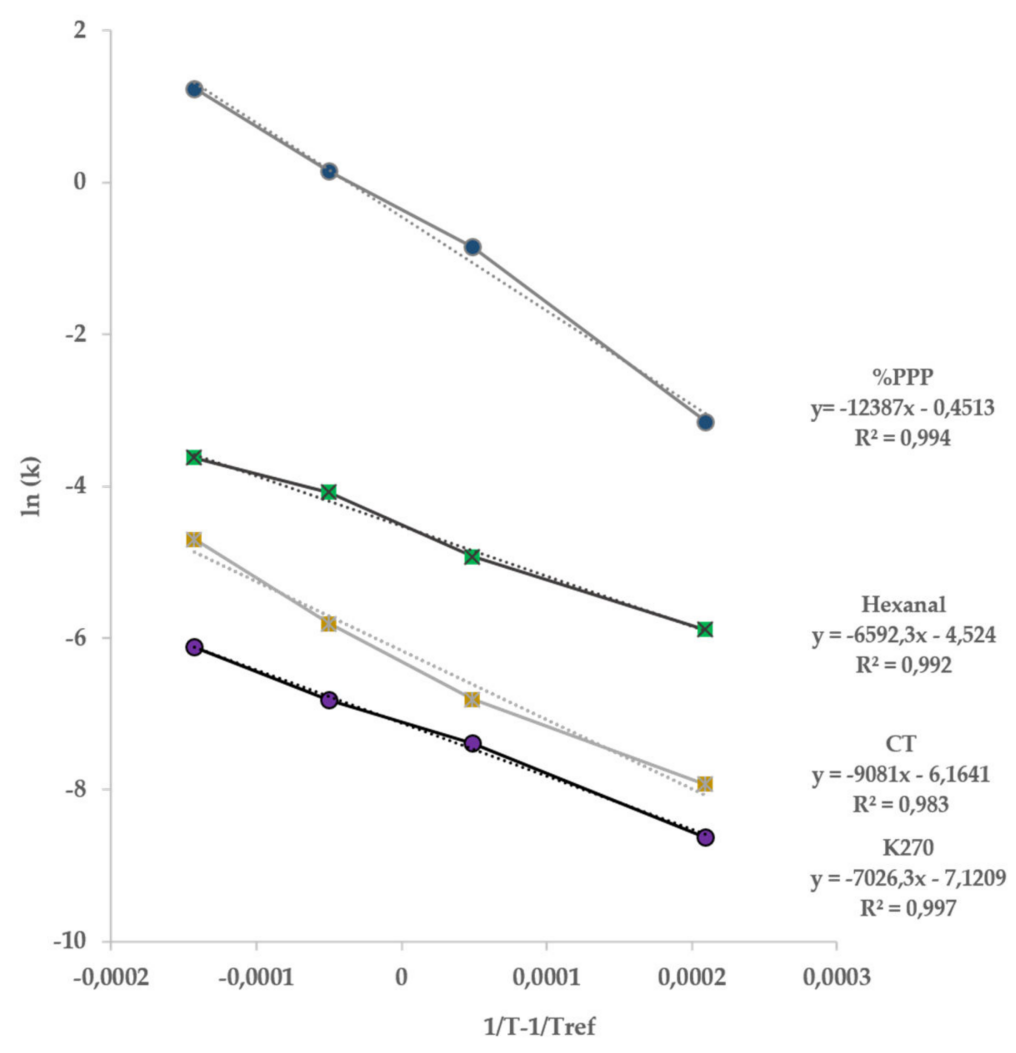

Figure 3. Arrhenius plot of apparent zero-order rate constants of K270, CT and \%PPP.

Table 3. Frequency factor $\left(k_{0}\right)$, activation energy $\left(E_{a}\right)$ and corresponding regression parameters of K270, $\mathrm{CT}$, hexanal and \%PPP in EVOO.

\begin{tabular}{cccc}
\hline Index & $\boldsymbol{k}_{\boldsymbol{o}}$ & $\boldsymbol{E}_{\boldsymbol{a}} \mathbf{( \mathbf { k J } / \mathbf { m o l } )}$ & $\boldsymbol{R}^{\mathbf{2}}$ \\
\hline $\mathrm{K}_{270}$ & $4.88 \times 10^{12}$ & 58.39 & 0.99 \\
$\mathrm{CT}$ & $6.57 \times 10^{9}$ & 75.46 & 0.99 \\
Hexanal & $9.28 \times 10^{10}$ & 54.78 & 0.99 \\
\%PPP & $1.30 \times 10^{17}$ & 102.94 & 0.99 \\
\hline
\end{tabular}




\subsection{Shelf-Life Estimation}

In the final part of the research, the Arrhenius equations acquired were used as predictive tools to estimate EVOO shelf-life at temperatures below $60^{\circ} \mathrm{C}$. To this aim, as described in the introduction, it is necessary to define a proper acceptability limit [30]. Among the indicators defined by EU regulation, the K270 limit was chosen the only one resulting applicable. This limit, equal to 0.22 (Table 1), represents the threshold value for the EVOO category [2,31]. On the other hand, no compulsory indications in the EU are available for other considered indexes. However, in consideration of the remarkable temperature sensitiveness of the \%PPP rate and being considered as freshness indicator by different authors [32,33], the estimation of SL was also carried out by using this indicator. An acceptability limit equal to $17 \%$ can be derived from the Australian Standard on EVOO [34]. The following equation was thus used to compute the product SL, Equation (4):

$$
\mathrm{SL}=\frac{I_{l i m}-I_{0}}{k_{T}}
$$

where $I_{0}$ is the initial value of the selected index, $I_{\text {lim }}$ is the value of the index defined as acceptability limit and $k_{\mathrm{T}}$ the rate constant at the temperature at which the SL would be defined. This value can be computed by using the Arrhenius equations reported in Figure 4.

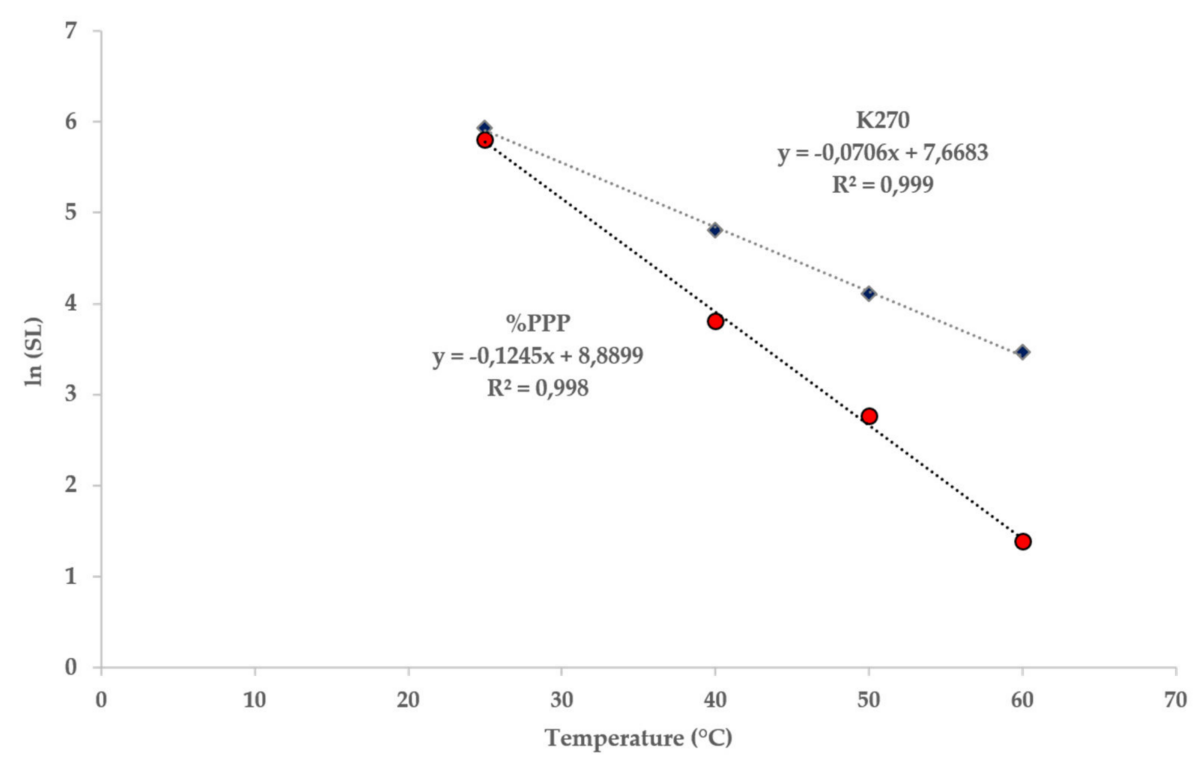

Figure 4. Shelf-life plots of EVOO by using K270 and \%PPP as shelf-life indicators.

By applying Equation (4), SL of EVOO was computed at different temperatures from 25 to $60^{\circ} \mathrm{C}$ (Table 4). It is interesting to note that the predicted SL at $25^{\circ} \mathrm{C}$ is comparable when using one of the two considered indices. However, as the temperature increased the expected product SL resulted considerably different and, in any case, shorter by considering \%PPP above $25^{\circ} \mathrm{C}$. As well known, $\%$ PPP has been proposed as a promising freshness indicator because pyropheophytin $a$ should be absent or present in trace amounts in freshly prepared EVOO oil [32,33].

The data were further used to generate the so-called shelf-life plot, reporting the $\ln (\mathrm{SL})$ as a function of storage temperature. As expected, a good linear relationship between these variables was acquired. The resulting regression lines can be considered the shelf-life predictive models that can be used to estimate EVOO lifetime at any temperature of interest. For instance, the expected SL at $20{ }^{\circ} \mathrm{C}$ is 544 and 601 days, considering K270 and \%PPP respectively. In this case, K270 represents the early indicator to predict the shelf-life. However, increasing the considered storage temperature, a reverse situation occurs with \%PPP becoming a more sensitive indicator. In fact, the SL computed with K270 resulted longer than that predicted by using \%PPP (265 days for K270 and 173 days for \%PPP). 
Table 4. Estimated shelf-life (days) at $25,40,50$ and $60^{\circ} \mathrm{C}$ by using $\mathrm{K} 270$ or $\% \mathrm{PPP}$ as quality indicators.

\begin{tabular}{ccc}
\hline \multirow{2}{*}{ Temperature $\left({ }^{\circ} \mathbf{C}\right)$} & \multicolumn{2}{c}{ Estimated Shelf-Life in Days (months) } \\
\cline { 2 - 3 } & K270 & \%PPP \\
\hline 25 & 377 & 332 \\
40 & 122 & 45 \\
50 & 61 & 16 \\
60 & 32 & 4 \\
\hline
\end{tabular}

\section{Conclusions}

In conclusion, results here reported demonstrated the feasibility of the ASLT methodology to develop a predictive tool for shelf-life prediction of EVOO. For well-packed products with reduced oxygen in headspace, we can conclude that nor primary oxidation products neither antioxidant content can be considered as good shelf-life indicators. At the moment, and based on EU regulation, the best SL index allowing to predict EVOO SL resulted K270. This means that by using this simple index and by knowing its temperature dependence, it would be possible to perform an accelerated test at $60^{\circ} \mathrm{C}$ able to estimate product shelf-life at ambient temperature in about 1 month.

Particularly interesting resulted also the possible exploitation of \% $\mathrm{PPP}$ as a freshness indicator. In fact, the changes of pyropheophytin $a$ were much more sensitive to temperature changes than the secondary oxidation indices. This parameter could be considered an early indicator of product performances on the market when the expected storage temperature of EVOO was higher than $25^{\circ} \mathrm{C}$.

The evaluation of conjugated fatty acids deriving from the oxidative process, which can be easily evaluated by HPLC, has been also shown to be an interesting new parameter.

From this promising starting point, further research is needed to validate and improve the robustness of the proposed approach by considering oils with different chemical characteristics. In fact, the understanding of the variability of the $E_{a}$ values and its relationship with EVOO chemical composition could allow generating a general SL model for EVOO.

Supplementary Materials: The following are available online at http://www.mdpi.com/2304-8158/9/3/295/s1, Table S1: Results obtained at $25^{\circ} \mathrm{C}$ storage condition for peroxide value, polyphenols, tocopherols and optical density at $232 \mathrm{~nm}$ at the relative storage times, Table S2: Results obtained at $40^{\circ} \mathrm{C}$ storage condition for peroxide value, polyphenols, tocopherols and optical density at $232 \mathrm{~nm}$ at the relative storage times, Table S3: Results obtained at $50{ }^{\circ} \mathrm{C}$ storage condition for peroxide value, polyphenols, tocopherols and optical density at $232 \mathrm{~nm}$ at the relative storage times, Table S4: Results obtained at $60^{\circ} \mathrm{C}$ storage condition for peroxide value, polyphenols, tocopherols and optical density at $232 \mathrm{~nm}$ at the relative storage times.

Author Contributions: Conceptualization, L.C., P.L., S.C. and M.C.N.; methodology, L.C., P.R., P.L., S.C. and M.C.N.; formal analysis, A.M., S.C. and P.L.; investigation, A.M.; resources, L.C., P.R., and L.P.; data curation, S.C., L.C., A.M., P.L. and M.C.N.; writing-original draft preparation, S.C., A.M. and P.L.; writing—review and editing, L.C., P.R. and M.C.N.; project administration, S.C. and P.L. All authors have read and agreed to the published version of the manuscript.

Funding: This research received no external funding.

Acknowledgments: The authors are grateful to Castel del Chianti (Tavarnelle Val di Pesa, Firenze, Italy) for their donation of oil samples.

Conflicts of Interest: The authors declare no conflict of interest.

\section{References}

1. Conte, L.; Bendini, A.; Valli, E.; Lucci, P.; Moret, S.; Maquet, A.; Lacoste, F.; Brereton, P.; García-González, D.L.; Moreda, W.; et al. Olive oil quality and authenticity: A review of current EU legislation, standards, relevant methods of analyses, their drawbacks and recommendations for the future. Trends Food Sci. Technol. 2019, in press. [CrossRef]

2. European Commission Regulation (EEC). No. 2568/91, On the Characteristics of Olive Oil and Olive-Residue Oil and on the Relevant Methods of Analysis. Available online: https://eur-lex.europa.eu/legal-content/EN/ TXT/?uri=CELEX\%3A01991R2568-20151016 (accessed on 15 January 2020). 
3. International Olive Oil Council. Trade Standard Applying to Olive Oil and Olive Pomace Oils. Available online: https://www.internationaloliveoil.org/wp-content/uploads/2019/12/trade-standard-REV-14-Eng.pdf (accessed on 15 January 2020).

4. Fadda, C.; Del Caro, A.; Sanguinetti, A.M.; Urgeghe, P.P.; Vacca, V.; Arca, P.P.; Piga, A. Changes during storage of quality parameters and in vitro antioxidant activity of extra virgin monovarietal oils obtained with two extraction technologies. Food Chem. 2012, 134, 1542-1548. [CrossRef] [PubMed]

5. Kotsiou, K.; Tasioula-Margari, M. Monitoring the phenolic compounds of Greek extra-virgin olive oils during storage. Food Chem. 2016, 200, 255-262. [CrossRef] [PubMed]

6. Gertz, C.; Fiebig, H.J. Pyropheophytin a: Determination of thermal degradation products of cholorohyll a in virgin olive oil. Eur. J. Lipid Sci. Technol. 2006, 108, 1062-1065. [CrossRef]

7. European Commission Regulation (EEC). No. 1169/2011 of the European Parliament and of the Council of 28 January 2002. Available online: https://books.google.com.hk/books?id= KVdxDwAAQBAJ\&pg=PA116\&lpg=PA116\&dq=European+Commission+Regulation+(EEC)+No. +1169/2011+of+the+European+Parliament+and+of+the+Council+of+28+January+2002\&source= bl\&ots=axNZoAuxLK\&sig=ACfU3U14dmKujTI6Nseo-altO3YtGYXW9A\&hl=zh-CN\&sa=X\&ved= 2ahUKEwiD4KWwr4DoAhXPc94KHYTjDWEQ6AEwA3oECAYQAQ\#v=onepage\&q=European \% 20Commission\%20Regulation\%20(EEC)\%20No.\%201169\%2F2011\%20of\%20the\%20European \%20Parliament \% 20and\%20of\%20the\%20Council\%20of\%2028\%20January\%202002\&f=false (accessed on 20 December 2019).

8. Nicoli, M.C. The shelf-life assessment process. In Shelf-life Assessment of Food; Nicoli, M.C., Ed.; CRC Press: Boca Raton, FL, USA, 2012.

9. Calligaris, S.; Manzocco, L.; Anese, M.; Nicoli, M.C. Shelf-life assessment of food undergoing oxidation-A review. Crit. Rev. Food Sci. Nutr. 2015, 56, 1903-1912. [CrossRef] [PubMed]

10. Calligaris, S.; Manzocco, L.; Anese, M.; Nicoli, M.C. Accelerated Shelf-Life Testing. Food Quality and Shelf-Life; Galanakis, C.M., Ed.; Academic Press: Cambridge, MA, USA, 2019.

11. Arrhenius, S.A. Textbook of the electrochemistry. In Larobok I Teoretisk Elektrokemi; Quando \& Handel: Leipzig, Germany, 1901.

12. Labuza, T.P.; Schmidl, M.K. Accelerated shelf-life testing of foods. Food Technol. 1985, 39, 57-62.

13. Corradini, M.G.; Peleg, M. Shelf-life estimation from accelerated data. Trends Food Sci. Technol. 2007, 18, 37-47. [CrossRef]

14. Mancebo-Campos, V.; Fregapane, G.; Salvador, M.D. Kinetic study for the development of an accelerated oxidative stability test to estimate virgin olive oil potential shelf-life. Eur. J. Lipid Sci. Technol. 2008, 110, 969-976. [CrossRef]

15. Calligaris, S.; Sovrano, S.; Manzocco, L.; Nicoli, M.C. Influence of crystallisation on the oxidative stability of extra virgin olive oil. J. Agric. Food Chem. 2006, 54, 529-535. [CrossRef] [PubMed]

16. Frankel, E.V. Stability methods. In Lipid Oxidation; Frankel, E.N., Ed.; The Oily Press: Sawston, Cambridge, UK, 2005.

17. Mizrahi, S. Accelerated shelf-life tests. In food and Beverage Stability and Shelf-Life; Kilcast, D., Subramaniam, P., Eds.; Woodhead Publishing Limited: Cambridge, UK, 2011.

18. International Olive Council Determination of Biophenols in Olive Oil by HPLC COI/T.20/Doc No. 29, Madrid. Available online: https://www.internationaloliveoil.org/wp-content/uploads/2019/11/COI-T.15-NC.-No-3Rev.-13-2019-Eng.pdf (accessed on 20 December 2019).

19. UNI 11702:2018. Determinazione Dell'idrossitirosolo e del Tirosolo Negli Oli Extra Vergini di Oliva. Available online: https://core.ac.uk/download/pdf/79621131.pdf (accessed on 20 December 2019).

20. ISO. Vegetable Fats and Oils: Determination of Degradation Products of Chlorophyll a and $a^{\prime}$ (Pheophytins $a, a^{\prime}$ and Pyropheophytins) (ISO 29841:2009); International Organization for Standardization: Geneva, Switzerland, 2009.

21. Rovellini, P.; Cortesi, N. Oxidative status of extra virgin olive oils: HPLC evaluation. Ital. J. Food Sci. 2004, 16, 335-344.

22. Vichi, S.; Castellote, A.I.; Pizzale, L.; Conte, L.S.; Buxaderas, S.; Lopez-Tamames, E. Analysis of virgin olive oil volatile compounds by headspace solid-phase microextraction coupled to gas chromatography with mass spectrometric and flame ionization detection. J. Chromatogr. A 2003, 983, 19-33. [CrossRef] 
23. EFSA. Panel on Dietetic Products Nutrition and Allergens Scientific Opinion on the substantiation of health claims related to polyphenols in olive and protection of LDL particles from oxidative damage (ID 1333, 1638 1639, 1696 2865), maintenance of normal blood HDL cholesterol concentrations (ID 1639), maintenance of normal blood pressure (ID 3781), “anti-inflammatory properties” (ID 1882), “contributes to the upper respiratory tract health" (ID 3468), "can help to maintain a normal function of gastrointestinal tract" (3779), and "contributes to body defences against external agents" (ID 3467) pursuant to Article 13(1) of Regulation (EC) No. 1924/2006. EFSA J. 2011, 9, 2033-2058.

24. Brenes, M.; Garcia, A.; Garcia, P.; Garrido, A. Acid hydrolysis of secoiridoid aglycons during storage of virgin olive oil. J. Agric. Food Chem. 2001, 49, 5609-5614. [CrossRef] [PubMed]

25. Gomes-Alonso, S.; Mancebo-Campos, V.M.; Salvador, M.D.; Fregapane, G. Evolution of major and minor components and oxidation indices of virgin olive oil during 21 months storage at room temperature. Food Chem. 2007, 1, 36-42. [CrossRef]

26. Kanavouras, A.; Hernandez-Mugnoz, P.; Coutelieris, F.; Selke, S. Oxidation-derivated flavor compounds as quality indicators for packaged olive oil. J. Am. Oil Chem. Soc. 2004, 81, 251-257. [CrossRef]

27. Van Boekel, M.A.J.S. Kinetic modelling of reactions in food: A critical review. Compr. Rev. Food Sci. Food Saf. 2008, 7, 144-158. [CrossRef]

28. Manzocco, L.; Calligaris, S.; Nicoli, M.C. Methods for food shelf-life determination and prediction. In Oxidation in Foods and Beverages and Antioxidant Applications; Decker, E.A., Elias, R.J., McClements, D.J., Eds.; Woodhead: Cambridge, UK, 2010.

29. Aparicio-Ruiz, R.; Minguez-Mosquera, M.I.; Gandul-Rojas, B. Thermal degradation kinetics of chlorophyll pigments in virgin olive oils. 1. Compounds of series a. J. Agric. Food Chem. 2010, 58, 6200-6208. [CrossRef] [PubMed]

30. Manzocco, L. The Acceptability Limit in Food Shelf-life Studies. Crit. Rev. Food Sci. Nutr. 2016, 56, 1640-1646. [CrossRef] [PubMed]

31. Commission Delegate Regulation (EU) No. 2015/1830. Available online: https://eur-lex.europa.eu/legalcontent/EN/TXT/?uri=celex:32015R1830 (accessed on 20 December 2019).

32. Australian Standards $® O$ Olive Oil and Olive-Pomace Oils. AS 5264-2011. Available online: https: //australianolives.com.au/wp-content/uploads/2019/05/Australian-Standards-P-Miller.pdf (accessed on 20 December 2019).

33. Aparicio-Ruiz, R.; Roca, M.; Gandul-Rojas, B. Mathematical model to predict the formation of Pyropheophytin $a$ in virgin olive oil during storage. J. Agric. Food Chem. 2012, 60, 7040-7049. [CrossRef] [PubMed]

34. Aparicio-Ruiz, R.; Tena, N.; Romero, R.; Aparicio, R.; Garcia-Gonzalez, D.L.; Morales, M.T. Predicting extra virgin olive oil freshness during storage by fluorescence spectroscopy. Grasas y Aceites 2017, 68. [CrossRef] 